\title{
Automatic Quantification of Changes in the Volume of Brain Structures
}

\author{
Guillaume Calmon $^{1}$, Neil Roberts ${ }^{1}$, Paul Eldridge ${ }^{2}$, and Jean-Philippe Thirion ${ }^{3}$ \\ 1 Magnetic Resonance and Image Analysis Research Centre (MARIARC), \\ University of Liverpool, UK \\ \{gcalmon, neil\}@liv.ac.uk \\ 2 Walton Centre for Neurology and Neurosurgery, Liverpool, UK \\ eldrid-pQwcnn.co.uk \\ 3 Épidaure Project, Institut National de Recherche en Informatique \\ et en Automatique (INRIA), Sophia Antipolis, France \\ thirion@epidaure.inria.fr
}

\begin{abstract}
We present an automatic technique to quantify changes in the volume of cerebral structures. The only manual step is a segmentation of the structure of interest in the first image. The image analysis comprises: i) a precise rigid co-registration of the time series of images, $i i$ ) the computation of residual deformations betweens pairs of images. Automatic quantification can be obtained either by propagation of the segmentation or by integration of the deformation field. These approaches have been applied to monitor brain atrophy in one patient and to investigate a 'mass effect' in tissue surrounding a brain tumour in four patients undergoing radiotherapy. Segmentation propagation gave good results for quantifying contrasted structures such as ventricles or wellcircumscribed tumours; however, integration of the deformations may be more appropriate to quantify diffusive tumours.
\end{abstract}

\section{Introduction}

The combination of Magnetic Resonance (MR) imaging and computer-based image analysis offers the opportunity for non-invasive measurement of changes in brain compartment volumes associated with a variety of diseases. Images obtained on successive occasions may be analysed separately, thanks to pointcounting technique [1], or repeated segmentations of the structure of interest. Alternatively, an increasingly popular approach involves first co-registering all the images obtained in the time series. Changes may be visualised by subtracting consecutive co-registered images in the time series [2], [3]. Quantification may be achieved by integrating small intensity changes within the boundary region $[4]$.

Furthermore, pathologies can be studied by comparison to a reference patient or model, thanks to non-rigid inter-patient registration. Several methods have been put forward, including the use of anatomical landmarks [5], [6] or differential features (e.g. edges, crest-lines or ridges). The possible output transformations 
include linear deformations [7], splines [8],[9], elastic models [10], [11], and viscous fluid models [12].

In this work, after an automatic rigid co-registration of succesive magnetic resonance (MR) images of a patient, a 3-D deformation field resulting from an intra-patient non-rigid registration is used to propagate an initial segmentation to each image in the time series. An alternative approach is to integrate the deformation field over a family of surfaces equidistant from the initial segmentation. The difference in the volume variation obtained by integration and by segmentation propagation may be accounted for by a mass effect in the surrounding tissue. A detailed description of the methods of segmentation propagation and deformation field integration are given in Sect. 2 below. In Sect. 3, we describe the application of segmentation propagation to a patient with primary progressive aphasia (PPA). In Sect. 4 we describe the application of both approaches to four patients with brain tumours undergoing radiotherapy.

\section{Quantification of Volume Variation}

\subsection{Overview of the Technique}

The technique comprises: $i$ ) a rigid co-registration of all the images in the series with the image acquired first, using an automatic detection of crest-lines in the images and a matching of their maxima of curvature [13]; ii) a non-rigid matching used to compute the residual deformations between successive pairs of images. Our implementation utilises the 'demons' algorithm [14], close to viscous-fluid models [15]. It outputs a 3-D vector field called $\mathbf{f}$. The only manual step of the technique is a (semi-automatic) segmentation of the structure of interest in the first image of the series. Automatic quantification can then be obtained either by propagation of the segmentation (see Sect. 2.2) or by integration of the deformation field $\mathbf{f}$ (see Sect. 2.3).

\subsection{Segmentation Propagation}

Given two images, $I_{1}$ and $I_{2}$, a segmentation $S_{1}$ in $I_{1}$, and assuming a regular grid $G$ lying in $I_{1}$, (see Fig. 1 , left), the number of nodes of $G$ within $S_{1}$, times the volume associated with a single node, is an approximation of the total volume $V\left(S_{1}\right)$. The number of nodes of $G$ within the deformed surface $\mathbf{f}\left(S_{1}\right)$ gives an approximation of $V\left(S_{2}\right)$, volume of the segmentation in the second image (Fig. 1, right). $V\left(S_{1}\right)$ is also the number of nodes of $\mathbf{f}(G)$ within $S_{2}=\mathbf{f}\left(S_{1}\right)$ (Fig. 1, centre), or, conversely, $V\left(S_{2}\right)$ is the number of nodes of $\mathbf{f}^{-1}(G)$ within $S_{1}$. This shows how we can efficiently compute $\Delta V_{\text {propag }}=V\left(S_{2}\right)-V\left(S_{1}\right)$ and resample $S_{1}$ into $S_{2}$ in a single pass over $I_{1}$, using the inverse deformation field $\mathbf{f}^{-1}$.

\subsection{Integration of the Deformation Field}

Motivations. Precise segmentation of a structure of interest is not always possible. A physical boundary to the tumour may not exist, due to the infiltration 
Image 1

Surface $S_{1}$ - Grid $G$

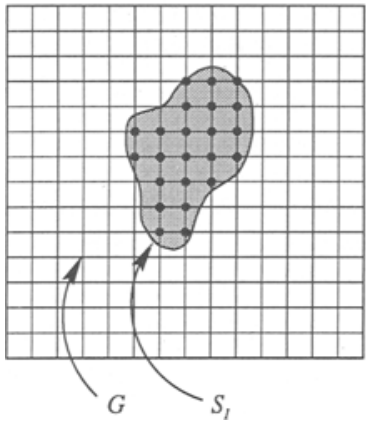

Surface $S_{2}$ - Deformed Grid f(G)

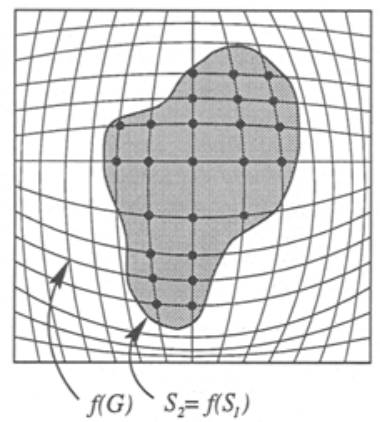

Image 2

Surface $\mathrm{S}_{2}-$ Grid $G$

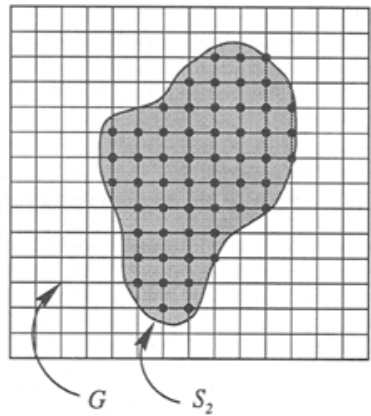

Fig. 1. Segmentation propagation: the volume variation is equal to the number of nodes (55) of the grid $G$ inside the segmentation $S_{2}$ in the second image minus the number of nodes (25) of $G$ inside the segmentation $S_{1}$ in the first image (which is the same as the number of nodes of the deformed grid $\mathbf{f}(G)$ inside the segmentation $S_{2}$ ). In this case, $\Delta V_{\text {propag }}=55-25$ voxels.

of tumourous cells through the healthy tissue. To quantify the pathological evolution of the tumour and its effects on the surrounding tissue more reliably, we have developped the following model (see also [16]):

Model for the Integration. The brain is divided into three components: tumour, healthy tissue, surrounding tissue. The 'tumour' is a (sometimes arbitrarily) delineated region in which we assume no brain function. The 'healthy tissue', is normal-looking tissue away from the tumour. Between these two, tissue surrounding the tumour can be a mix of tumourous cells, edema, brain tissue... To monitor volume variation in surrounding tissue, a family of surfaces parallel

Integration domaine

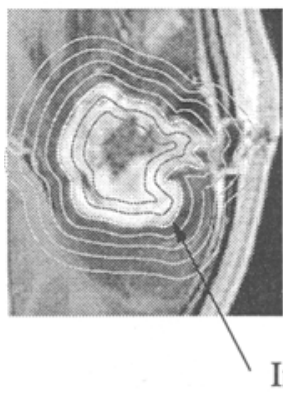

\section{Volume Variation Profile}

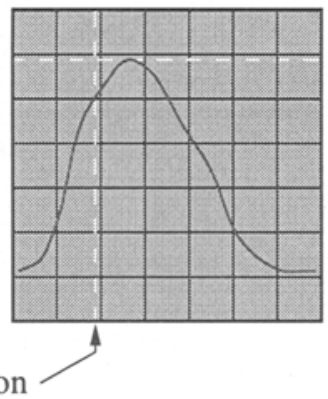

$\Delta V_{\text {integ }}$

Initial Segmentation

Fig. 2. Integration of the deformation over embedded surfaces yields a profile of the volume variation. 
to the surface of the original segmentation is computed (see Fig. 2, left). Each of these surfaces yields a value of volume variation, giving a profile $\Delta V$ against the index $i$ of the corresponding surface. It starts from zero, and by thresholding $\mathbf{f}$, is forced to zero when we reach the limit of the integration domain. $\Delta V_{\text {integ }}$ is the maximum or minimum of the profile.

Practical Computation of the Profile. From now on, we call $G$ and $S$ the grid and surface, respectively, and assume that we have a family of embedded surface $S^{(i)}(i \in[-m \ldots m])$ surrounding $S$. This can be efficiently achieved by computing a 3-D distance map, for example using the chamfer distance, in which the enclosed surfaces $S^{(i)}$ are defined by an intensity level $\left(S^{(0)}\right.$ corresponding to $S$ ). Suppose that we have defined a region of interest (ROI) containing $n$ grid nodes of $G$ :

- We define two arrays of numbers $\left\{M_{i}^{G}\right\}$ and $\left\{M_{i}^{\mathbf{f}(G)}\right\}, i \in[-m \ldots m]$.

- For the $n$ nodes of $G, P_{j}(x, y, z)(j \in[0 . . n])$, we determine the index $i$ of the shell delimited by the surfaces $S^{(i)}$ and $S^{(i+1)}$ which contains $P_{j}$ (resp. $\mathbf{f}\left(P_{j}\right)$ ). The index $i$ can be obtained in constant time with the distance map described above For each $P_{j}$, we increment the corresponding bucket $M_{i}^{G}$ (resp. $M_{i}^{\mathbf{f}(G)}$ ).

- Next, we compute incrementally the arrays

$$
N_{i}^{G}=\sum_{k=0}^{i-1} M_{k}^{G} \text { and } N_{i}^{\mathrm{f}(G)}=\sum_{k=0}^{i-1} M_{k}^{\mathbf{f}(G)} .
$$

$N_{i}^{G}$ (resp. $N_{i}^{\mathbf{f}(G)}$ ) represents the total number of nodes of $G$ (resp. $\mathbf{f}(G)$ ) within $S^{(i)}$.

- Finally, the volume variation profile is $\Delta V_{i}=\left(N_{i}^{G}-N_{i}^{\mathbf{f}(G)}\right) \times V($ voxel $)$ against $i, i \in[-m \ldots m]$.

\subsection{Definition of the 'Mass Effect' in Surrounding Tissue}

Segmentation propagation and deformation field integration represent two different ways of automatically quantifying the variations in the volume of a structure of interest. If we call $V_{1}$ (resp. $V_{2}$ ) the volume of the tumour in the initial (resp. final) image, and $V_{1}+v_{1}$ (resp. $V_{2}+v_{2}$ ) the volume of tumour plus surrounding tissue, then $\Delta V_{\text {propag }}$ is a quantification of $V_{2}-V_{1}$, whereas $\Delta V_{\text {integ }}$ is a (more imprecise) estimation of $\left(V_{2}+v_{2}\right)-\left(V_{1}+v_{1}\right)$. To monitor brain response to the treatment and/or to tumour evolution, we introduce the 'mass effect' as

$$
\frac{v_{2}-v_{1}}{\Delta t}=\frac{\Delta V_{\text {integ }}-\Delta V_{\text {propag }}}{\Delta t},
$$

where $\Delta t$ is the time gap between the two acquisitions. 


\section{Quantification of Ventricular Enlargement in Primary Progressive Aphasia}

The application of segmentation propagation to monitor the increase in the volume of the lateral ventricles in a patient with primary progressive aphasia (PPA) is examined. We compared the rate of ventricular enlargement to the estimation of the rate of whole brain atrophy obtained by stereology.

\subsection{Subject and MRI}

A 65 year old male with a diagnosis of PPA was scanned using 1.5 T SIGNA whole body MR imaging system (General Electric, Milwaukee, USA). High resolution T1-weighted SPGR images were obtained on four separate occasions approximately one year apart. The images, of an initial resolution of $0.78 \times 0.78 \times 1.6$ $\mathrm{mm}^{3}$ for $256 \times 256 \times 124$ voxels were sinc-interpolated to an array of $200 \times 200 \times 199$ isotropic (i.e. cubic) voxels of side $1.0 \mathrm{~mm}$.

\subsection{Segmentation of Left and Right Ventricles}

Segmentation of the left and right lateral ventricles (LV and RV) was achieved by interactively thresholding the image and classifying connected components
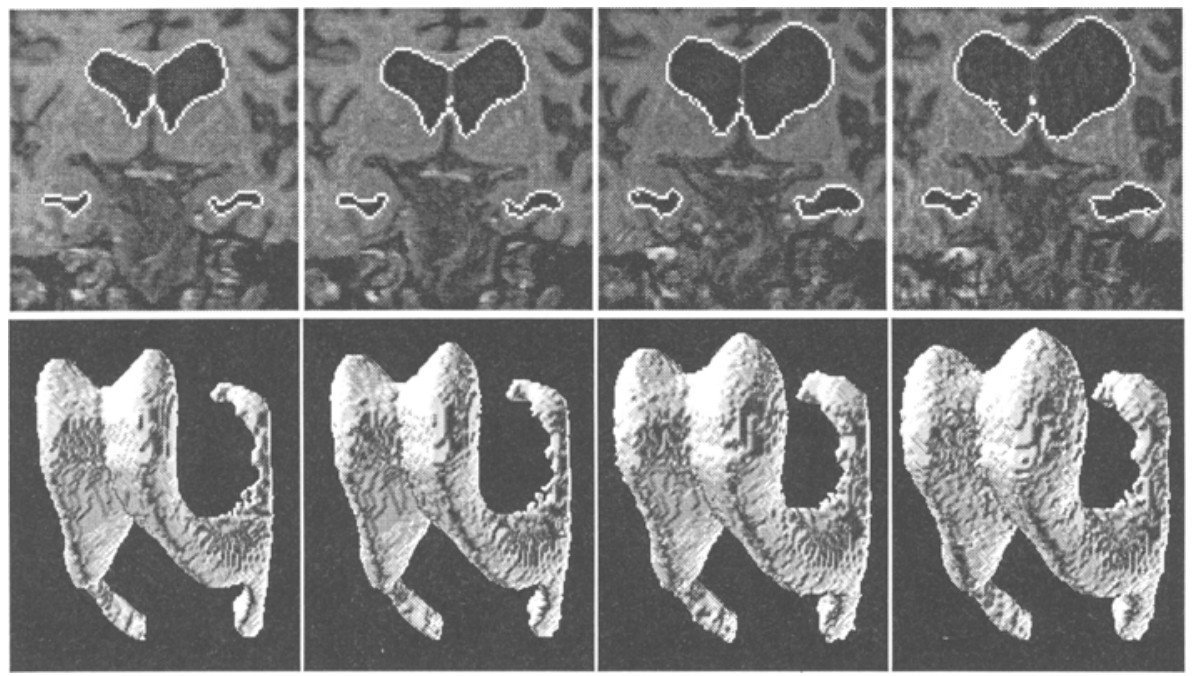

Fig. 3. Segmentation of the lateral ventricles in $4 \mathrm{MR}$ images of a patient with brain atrophy, approximately one year appart. Top, from left to right: coronal sections in successive time points. Bottom: 3 -D rendering of the lateral ventricles (images have been flipped to reflect upper coronal sections).

of the eroded binary image. Minimal manual editing was necessary to cut the 
lateral ventricles from the third and fourth ventricles. We manually partitioned the left and right ventricles to separately compare volume variations in the left and right hemisphere.

\subsection{Results}

The rate of increase of volume of the left (resp. right) ventricle as measured by image analysis was $33.0 \mathrm{~mm}^{3}$ per day (resp. $17.3 \mathrm{~mm}^{3}$ per day) compared with the findings of stereology: $31.3 \mathrm{~mm}^{3}$ per day (resp. $15.5 \mathrm{~mm}^{3}$ per day); linear regression relationship $R>0.952$ in all cases (see Fig. 4). Corresponding values for the rate of decrease of left (resp. right) cerebral hemisphere volume obtained using stereology was $-137.9 \mathrm{~mm}^{3}$ per day (resp. $-124.2 \mathrm{~mm}^{3}$ per day); linear regression relationship $R>0.88$.

(a)

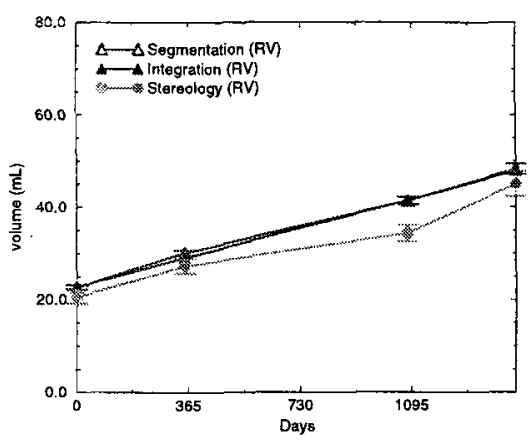

(b)

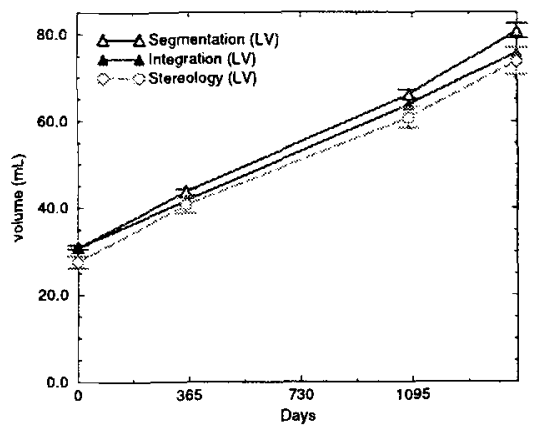

Fig. 4. Patient with brain atrophy. Variation of volume of (a) right and (b) left ventricles over a period of four years

\subsection{Discussion}

Results of automatic segmentation propagation are consistent with those obtained by stereology. The larger rate of increase of volume observed for the left ventricle is consistent with a preferential decrease in the volume of the left cerebral hemisphere associated with the loss of language. Segmentation propagation represents a reliable and sensitive technique for monitoring and quantifying ventricular enlargement.

\section{Temporal Changes in the Volume of Cerebral Tumours Following Radiotherapy}

We investigated changes in brain tumours and lateral cerebral ventricles in four patients undergoing radiotherapy (RT). We computed the variation in the volume of the tumour and ventricles over time, and tried to quantify the 'mass effect' in the brain tissue surrounding the tumour. 


\subsection{Subjects and MRI}

Four patients with surgically confirmed high grade astrocytoma were imaged on an average of 5 occasions. The patients gave informed written consent of their willingness to participate in the study, which was approved by the local Ethics Committee. For each patient, prior to and approximately monthly after RT (and brachytherapy in the case of patient $C$ ), T1-weighted 3-D MR IRPREP images have been obtained following intravenous injection of the contrast agent GdDTPA. The resolution of the $256 \times 256 \times 124$ acquired voxels is $0.78 \times 0.78 \times 1.6$ $\mathrm{mm}^{3}$.
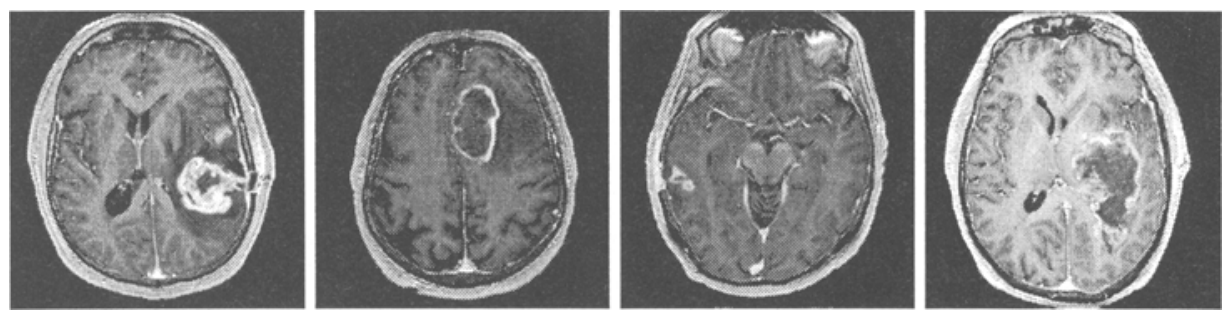

Fig. 5. Glioma study: from left to right, patient $A, B, C, D$.

\subsection{Segmentation of Structures of Interest}

We used the same approach to segment the lateral ventricles as described in Sect. 3.2. In patients $A$, the disruption of the blood-brain barrier produced a conspicuous ring of enhancement around the tumour, so that segmentation could be achieved by thresholding followed by minimal manual editing. Patient $C$ had a small, well contrasted tumour, for which the same procedure applied. In patient $B$ and $D$, the tumour margins were more diffuse and more approximate segmentations were obtained. Nevertheless, for all patients, the effect of therapy could be quantified.

\subsection{Results and discussion}

Segmentation propagation proved useful for the monitoring of volume changes in the lateral ventricles following administration of RT to cerebral tumours (see Table 1). The approach also proved appropriate for studying changes in volume of tumours with enhanced boundaries (patients $A$ and $C$ ). However, integration of the deformation field represented a useful approach for quantifying changes in volume of tumours in patients $B$ and $D$. In addition, it enabled quantification of changes in tissue surrounding the tumour.

In patients $A$ and $B$, renewed growth of the tumour approximately 100 days after RT corresponded to a to a decrease in the rate of ventricular enlargement. The brain tissue surrounding the tumour expanded by between $60 \mathrm{~mm}^{3}$ per day 
Table 1. Ventricles and tumours volumes and surrounding tissue mass effect in four patients with brain tumour.

\begin{tabular}{|ll|c|c|c|c|}
\hline \multicolumn{2}{|c|}{ Patients } & $A$ & $B$ & $C$ & $D$ \\
\hline Ventricles (ml) & before RT & 26.3 & 13.6 & 25.3 & 15.7 \\
& after RT & 30.3 & 15.1 & 31.9 & 27.5 \\
\hline \multirow{3}{*}{ Tumour (ml) } & before RT & 23.6 & 36.4 & 1.4 & 62.3 \\
& after RT & 19.2 & 32.1 & 0.85 & 50.8 \\
& regrowth & 33.3 & 36.3 & n/a & 62.1 \\
\hline Mass effect & after RT & +20 & -80 & -40 & -200 \\
$\left(\mathrm{~mm}^{3}\right.$ per day) & regrowth & +60 & +80 & n/a & +130 \\
\hline
\end{tabular}

in patient $A$, and $80 \mathrm{~mm}^{3}$ per day in patient $B$. In patient $C$, brachytherapy at day 49 induces an enlargement of the tumour, followed by a shrinkage between days 70 and 130 . Negative mass effect occurs after brachytherapy $\left(-30 \mathrm{~mm}^{3}\right.$ per day). In patient $D$, the tumour decreased in volume over a period of at least 100 days following radiotherapy, during which time the lateral ventricles enlarged. However, the tumour eventually regrew. The mass effects during tumour shrinkage $\left(-200 \mathrm{~mm}^{3}\right.$ per day) and renewed growth $\left(120 \mathrm{~mm}^{3}\right.$ per day) are larger than in all the other patients.

\section{Conclusion}

Non-rigid registration of 3-D MR images obtained on successive occasions is a reliable and sensitive technique for monitoring and quantifying changes in volume in brain structures. Results are consistent with those obtained by stereology. Furthermore, analysis of the deformation field may give an estimation of tissue expansion or compaction in a region of interest. In the case where the region of interest is the exact segmentation of a structure of interest (e.g. the lateral ventricles), accurate changes in volume can be automatically quantified by segmentation propagation.

\section{Acknowledgments}

We gratefully acknowledge the support of our partners, Dr. M. Doran and Dr. A. Brodbelt from the Walton Centre for Neurology and Neurosurgery, Liverpool, UK, and Dr. B. Jones from the Clatterbridge Centre for Oncology, Wirral, UK. We also wish to thank Ms. J. Webb and Mr. Q. Gong, from MARIARC, who performed the stereological measurements, and performed the scanning of the patients. 


\section{References}

1. N. Roberts, A. S. Garden, L. M. Cruz-Orive, G. H. Whitehouse, and R. H. T. Edwards. Estimation of fetal volume by MR imaging and stereology. Bristish Journal of Radiology (BJR), 67:1067-1077, 1994.

2. Joseph V. Hajnal, Nadeem Saeed, Angela Oatridge, Elaime J. Williams, Ian R. Young, and Graeme M. Bydder. Detection of subtle brain changes using subvoxel registration and subtraction of serial MR images. Journal of Computer Assisted Tomography (JCAT), 19(5):677-691, 1995.

3. Louis Lemieux. The segmentation and estimation of noise in difference images of co-registered MRI scan pairs. In Medical Image Understanding and Analysis (MIUA'97), Oxford, UK, July 1997.

4. Peter A. Freeborough, Roger P. Woods, and Nick C. Fox. Accurate registration of serial 3D MR brain images and its application to visualizing change in neurodegenerative disorders. Journal of Computer Assisted Tomography (JCAT), 20(6):10121022, 1996.

5. D. Lemoine, C. Barillot, B. Gibaud, and E. Pasqualini. An anatomical-based 3D registration system of multimodality and atlas data in neurosurgery. In Lecture Notes in Computer Science, volume 511, pages 154-164, 1991.

6. Jean Talairach and Pierre Tournoux. Referentially oriented cerebral MRI anatomy. Thieme Medical Publishers, New York, United-States, 1993.

7. D. Louis Collins, Peter Neelin, Terrence M. Peters, and Allan C. Evans. Automatic 3D intersubject registration of $\mathrm{MR}$ volumetric data in standardized Talairach space. Journal of Computer Assisted Tomography (JCAT), 18(2):192-205, 1994.

8. Fred L. Bookstein. Principal warps: Thin-plate splines and the decomposition of deformations. IEEE Transactions in Pattern Analysis and Machine Intelligence (PAMI), 11(6):567-585, June 1989.

9. J. Declerck, G. Subsol, Jean-Philippe Thirion, and N. Ayache. Automatic retrieval of anatomical structures in 3D medical images. In Computer Vision, Virtual Reality and Robotics in Medicine, CVRMed '95, pages 153-162, Nice, France, April 1995.

10. R. Bajcsy and S. Kovačič. Multiresolution Elastic Matching. Computer Vision, Graphics and Image Processing, 46:1-21, 1989.

11. Jim C. Gee, Martin Reivich, and Ruzena Bajcsy. Elastically deforming 3D atlas to match anatomical brain images. Journal of Computer Assisted Tomography (JCAT), 17(2):225-236, 1993.

12. G. E. Christensen, R. D. Rabbitt, and M. I. Miller. 3D brain mapping using a deformable neuroanatomy. Physics in Medicine and Biology, 39:609-618, 1994.

13. Jean-Philippe Thirion. New feature points based on geometric invariants for $3 D$ image registration. International Journal of Computer Vision, 18(2):121-137, 1996.

14. Jean-Philippe Thirion. Non-rigid matching using demons. In Computer Vision and Pattern Recognition, San Francisco, California USA, June 1996.

15. Morten Bro-Nielsen and Claus Gramkow. Fast fluid registration of medical images. In Visualization in Biomedical Computing (VBC'96), pages 267-276, Hamburg, Germany, September 1996.

16. Jean-Philippe Thirion and Guillaume Calmon. Measuring lesion growth from 3D medical images. In IEEE Non Rigid and Articulated Motion Workshop in conjunction with CVPR'97, pages 112-119, Puerto Rico, USA, June 1997. also available as technical report RR-3101 at $\mathrm{ftp}$.inria.fr/INRIA/tech-reports/RR. 